\title{
Differential diagnostic value of 64-slice spiral computed tomography in solitary pulmonary nodule
}

\author{
XIAOMING WANG ${ }^{1}$, LIANG LV ${ }^{1}$, QINYUN ZHENG ${ }^{2}$, XIANLONG HUANG $^{1}$ and BIQIANG LI ${ }^{1}$ \\ Departments of ${ }^{1}$ Radiology and ${ }^{2}$ Physical Examination Center, The General Chongqing Hospital, \\ Chongqing 400014, P.R. China
}

Received June 25, 2017; Accepted January 16, 2018

DOI: $10.3892 / \mathrm{etm} .2018 .6041$

\begin{abstract}
The present study aimed to evaluate the diagnostic value of 64-slice spiral multivariate computed tomography (CT) combined with dynamic contrast-enhanced scanning for benign and malignant solitary pulmonary nodules (SPNs). A total of 93 patients with SPN as diagnosed by CT were included. All these patients were subjected to routine and dynamic enhancement $\mathrm{CT}$ scanning. After reconstruction, the morphological characteristics following dynamic enhancement were analyzed, and compared for the benign and malignant SPN cases. The incidences of lobulation, spicular sign, pleural indentation and vacuole sign in the malignant SPN group were significantly higher compared with the benign SPN group. During the dynamic enhancement scanning, the $\mathrm{CT}$ values at all the time points for the inflammatory and malignant SPN groups were significantly higher than the benign SPN group. No significant differences were observed in the dynamic enhancement $\mathrm{CT}$ values at 30,60, 90 and $120 \mathrm{sec}$ between the inflammatory, and malignant SPN groups. However, in the inflammatory SPN group, the dynamic enhancement CT values at 300 and $540 \mathrm{sec}$ were significantly lower than the malignant SPN group. Notably, the diagnostic accordance rate for the morphological signs combined with dynamic enhancement diagnosis was significantly higher than the morphological signs alone. The 64-slice spiral CT morphological signs combined with dynamic enhancement detection can be more effective for the differential diagnosis of benign and malignant SPN, which may provide potent evidence for the early clinical treatment.
\end{abstract}

Correspondence to: Dr Liang Lv, Department of Radiology, The General Chongqing Hospital, 104 Pipashan Zheng Street, Chongqing 400014, P.R. China

E-mail: lvliang1819@163.com

Dr Qinyun Zheng, Department of Physical Examination Center, The General Chongqing Hospital, 104 Pipashan Zheng Street, Chongqing 400014, P.R. China

E-mail: huhujjkk322@sina.com

Key words: solitary pulmonary nodules, computed tomography, differential diagnosis, dynamic enhancement

\section{Introduction}

Solitary pulmonary nodule (SPN) represents solitary round lesions with diameter of less than $3.0 \mathrm{~cm}$, which are generally not accompanied by nodular lesions (such as atelectasis, lymphadenopathy, and pleural effusion) (1). Common causes for SPN mainly include tumor-like lesions (benign and malignant lesions), infectious lesions, and non-infectious lesions. There are significant differences in the treatment and prognosis between benign and malignant lesions. Therefore, the diagnosis of benign and malignant lesions is of great importance for the disease clinical diagnosis and treatment $(2,3)$. However, differential diagnosis of SPN (benign or malignant) is still an important and difficult task for imaging technology (4).

In recent years, the rapid development of multislice spiral computed tomography (MSCT) technology represents a promising approach for the diagnosis of SPN. MSCT can display the lesions more clearly and intuitively, which is great helpful for the qualitative diagnosis of SPN (5). Moreover, MSCT dynamic enhancement scanning could provide more detailed evidence for the blood supply of lesions and their relationship with surrounding blood vessels, which is a reliable basis for the clinical feasibility of surgery (6). In this study, SPN was systematically characterized with the combination of dynamic enhancement parameters and morphological features of nodules, in order to improve the correct diagnosis rate of the disease.

\section{Materials and methods}

Study subjects. Totally 93 patients with SPN, who were confirmed by CT detection in our hospital from July 2014 to June 2016, were included in this study. There were 55 males and 38 females, with an average age of $40.71 \pm 3.64$ years (ranging from 27 to 61 years). According to the pathological detection, 52 cases were ultimately diagnosed as malignant nodules and 41 cases were finally determined as benign nodules (including 26 cases of inflammatory nodules).

Inclusion criteria were as follows: i) Patients of SPN as confirmed by CT detection, with diameter of $<30 \mathrm{~mm}$, without atelectasis, satellite lesions, or lymphadenopathy (mediastinal lymph node, or in the hilus pulmonis region); ii) patients with detailed, complete clinical data, as well as corresponding 
examination results; iii) patients without serious organic diseases of heart, brain, and other vital organs; and iv) patients not receiving radiotherapy or chemotherapy. Prior written and informed consent were obtained from every patient and the study was approved by the ethics review board of our hospital. Exclusion criteria included: i) Patients with multiple pulmonary nodules, or with lung mass with diameter of $>30 \mathrm{~mm}$; ii) patients with contrast agent allergies; iii) patients who could not tolerate imaging due to failures of heart, liver, kidney, and/or other organs; and iv) patients who refused to participate in this study.

CT scanning. CT scanning was performed with the 64-slice multidetector CT scanner (Aquilion 64; Toshiba, Otawara, Japan). All patients were subjected to routine and enhanced CT scanning. During scanning, the patients were asked to hold their breath after breathing in. Scanning range covered from the apex pulmonis to the basis pulmonis. CT scanning parameters were as follows: Voltage, $120 \mathrm{kV}$; current, $200 \mathrm{mAs}$; slice thickness, $3.0 \mathrm{~mm}$; pitch, $1.5 \mathrm{~mm}$; and reconstruction interval, $3.0 \mathrm{~mm}$. Based on the routine scanning, dynamic enhancement scanning was conducted over the reduced range covering the nodules $( \pm 2 \mathrm{~cm})$, with the same scanning parameters mentioned. Enhanced scanning contrast agent was iohexol $(100 \mathrm{ml})$, which was injected (as a bolus) with the high-pressure syringe, at the injecting rate of $2 \mathrm{ml} / \mathrm{s}$. Scanning images were obtained at $0,30,60,90,120,300$ and $540 \mathrm{sec}$, respectively, after the injection of contrast agent.

Image processing and analyzing. For the original images from $\mathrm{CT}$ routine and dynamic enhancement scanning, 0.625-mm thin-slice reconstruction was conducted. Obtained data were transferred to the GE ADW 4.3 post-processing work station (General Electric Healthcare, Milwaukee, WI, USA), and multi-planar reformation (MPR), curved planar reformation (CPR), and volume reproduction (VR) were performed. The lesions were clearly displayed, particularly for the SPN size, distribution, morphology, and boundary, as well as the relationship between SPN and bronchus. The mean CT values for each time point from the routine and dynamic enhancement scanning were obtained. MSCT original scanning images, reconstruction images, and dynamic enhancement images were analyzed by two senior radiologists. Each case was diagnosed, and the lesion location, range, and bronchial involvement were determined. Diagnosis was confirmed only when consensus was achieve.

Statistical analysis. Data were expressed as mean $\pm \mathrm{SD}$. SPSS 14.0 software (SPSS, Inc., Chicago, IL, USA) was used for statistical analysis. Measurement data were analyzed by ANOVA, with the LSD test, while counting data were compared with the $\chi^{2}$ test. $\mathrm{P}<0.05$ was considered to indicate a statistically significant difference.

\section{Results}

Imaging characteristics of benign and malignant SPN cases. All these 93 patients were diagnosed by biopsy or surgical pathology. Detailed results from the pathological diagnosis were shown in Table I. For the CT scanning, the main signs
Table I. Pathological diagnosis of 93 included patients.

\begin{tabular}{lrc}
\hline Diagnosis & No. & Incidence $(\%)$ \\
\hline Malignant SPN & 52 & 55.91 \\
Squamous carcinoma & 25 & 48.08 \\
Adenocarcinoma & 17 & 32.69 \\
Bronchial alveolar carcinoma & 7 & 13.46 \\
Small cell carcinoma & 3 & 5.77 \\
Benign SPN & 41 & 44.09 \\
Sclerosing hemangioma & 6 & 14.63 \\
Hamartoma & 5 & 12.20 \\
Tuberculosis ball & 11 & 26.83 \\
Inflammatory nodules & 19 & 46.34 \\
\hline
\end{tabular}

SPN, Solitary pulmonary nodule.

for these 93 SPN cases included the lobulation, spicular sign, pleural indentation, vessel convergence, vacuole sign, aerial bronchogram, satellite lesions, and calcification (Fig. 1). As shown in Table II, compared with the benign SPN group, the incidences of lobulation, spicular sign, pleural indentation, and vacuole sign were significantly elevated in the malignant SPN group $(\mathrm{P}<0.05)$. On the other hand, the incidence of calcification in the benign SPN group was significantly higher than the malignant SPN group $(\mathrm{P}<0.05)$. However, no significant differences were observed in the incidences of vessel convergence, aerial bronchogram, or satellite lesions between the benign and malignant SPN groups $(\mathrm{P}>0.05)$. Taken together, these results suggest that significant differences in the $\mathrm{CT}$ image morphology could be observed between benign and malignant SPN cases.

Dynamic enhancement of different SPN types. Different time density curve (TDC) types were observed for malignant and benign SPN, and inflammatory nodules (Fig. 2). Our results showed that, for the malignant SPN, obvious enhancement was observed at $30 \mathrm{sec}$ after contrast injection, which peaked at $90 \mathrm{sec}$ after injection and then maintained at high levels for a period (Fig. 3). For the benign SPN, only mild or less evident enhancement was noted during the enhancement process. For the inflammatory nodules, obvious enhancement was observed immediately after the contrast injection, which begun to decline at $120 \mathrm{sec}$ after injection (Fig. 4). For the malignant SPN and inflammatory nodules, during the dynamic enhancement scanning, the CT values at all the time points were significantly higher than the benign SPN group $(\mathrm{t}=8.10$, $8.55,8.32,8.97,9.44,10.52,7.62,7.85,6.02,5.41,5.22$, and 3.69 , respectively; $\mathrm{P}<0.05)$. Dynamic enhancement $\mathrm{CT}$ values at different time points between benign and malignant groups were next compared. Our results showed that, dynamic enhancement CT values were elevated for both groups, at 30, 60,90 , and $120 \mathrm{sec}$ after contrast injection, without significant difference ( $\mathrm{t}=3.27$ and 3.69, respectively; $\mathrm{P}<0.05)$. However, at 300 and $540 \mathrm{sec}$ after contrast injection, the dynamic enhancement CT values for the inflammatory nodule group was obviously declined, which were significantly lower than 

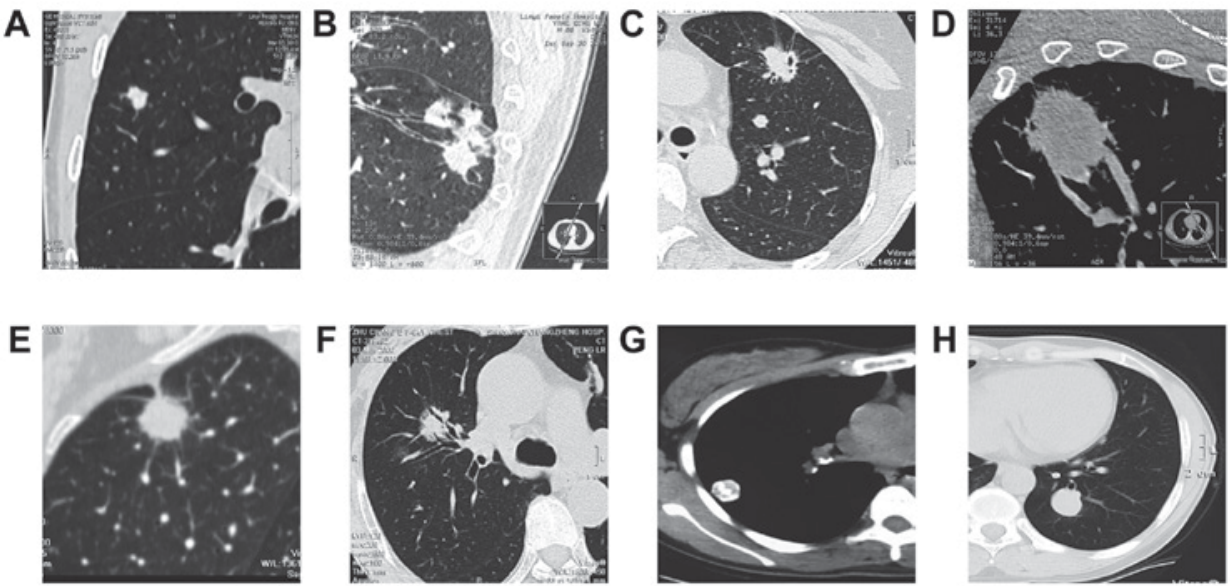

Figure 1. Imaging characteristics of benign and malignant SPN cases. (A) MPR reconstruction showing right upper lobe nodule with shallow lobulation (adenocarcinoma as confirmed by postoperative pathology). (B) MPR reconstruction showing right lower lobe nodule with deep lobulation. Pleural indentation and vacuole sign were also observed, and the bronchus was truncated at the nodule boundary (squamous carcinoma as confirmed by postoperative pathology). (C) Left upper lobe with lobulation, spicular sign at the boundary. Vacuole sign was also observed (adenocarcinoma as confirmed by postoperative pathology). (D) MPR reconstruction showing that the left upper lobe nodules, surrounded by a number of blood vessels accumulating towards the nodule, i.e., vessel convergence (squamous carcinoma as confirmed by postoperative pathology). (E) MPR reconstruction showing right upper lobe nodule, with spicular sign and pleural indentation (adenocarcinoma as confirmed by postoperative pathology). (F) MPR reconstruction showing right upper lobe nodule, with vacuole sign inside (squamous carcinoma as confirmed by postoperative pathology). (G) Right lower lobe nodule, with smooth boundary and calcification sign inside (hamartoma as confirmed by puncture biopsy). (H) Left lower lobe nodule, with smooth boundary and uniform density, closely related to peripheral blood vessels (sclerosing hemangioma as confirmed by postoperative pathology).

the dynamic enhancement $\mathrm{CT}$ values at corresponding time points in the malignant SPN group ( $\mathrm{t}=3.27$ and 3.69; $\mathrm{P}<0.05)$. In fact, no obvious decline was observed in the dynamic enhancement CT value in the malignant SPN group, at 300 and $540 \mathrm{sec}$ after contrast injection (Table III). Taken together, these results suggest that, during the dynamic enhancement, significant differences could be observed in the CT values between different TDCs and different time points.

Diagnostic accordance rate of morphological signs combined with dynamic enhancement diagnosis. Diagnostic accordance rates of morphological signs alone, as well as morphological signs combined with dynamic enhancement diagnosis, were then investigated. Our results showed that, the diagnostic accordance rate for the morphological signs combined with dynamic enhancement diagnosis was $83.87 \%$, which was significantly higher than that for the morphological signs alone (70.97\%) (Table IV; $\mathrm{P}<0.05$ ). These results suggest that, CT image morphology combined with dynamic enhancement diagnosis might lead to relatively high diagnostic accordance rate.

\section{Discussion}

SPN have been observed for different lesions. Malignant SPN cases are mostly peripheral lung cancers, with less cases of metastasis. Benign SPN cases mainly include tuberculoma, inflammatory lesions, and hamartoma (7). Qualitative diagnosis of SPN is important for the clinical treatment and disease prognosis. However, there are many difficulties for the CT-based qualitative diagnosis of SPN in clinic due to it simply depends on the morphological changes $(1,8)$. With the rapid development and wide application of CT technology, more and more SPN cases have been diagnosed. Therefore, the early detection and accurate diagnosis of SPN are critical and important for the clinical treatment and disease.
Thoracic CT 3-D reconstruction is superior to ordinary thoracic CT scanning in the diagnostic sensitivity and the morphological observation of SPN. For the 3-D reconstruction, due to its high resolution and satisfactory continuity, small lesions would not be missed, and it is easier to determine the lesion size and accurate location. Moreover, the surface characteristics of SPN (lobulation, sub-thorns, thorn-like processes, and halo sign) could be intuitively displayed, which might contribute to distinguish the benign and malignant cases, and help to develop the therapeutic regimen. Compared with the 16-slice MSCT, the 64-slice MSCT has more powerful post-processing ability, including MPR, CPR, MIP, and VRT, which could clearly show the nodule morphology and various signs. Meanwhile, scanning speed has been greatly increased, which reduces the respiratory motion-induced artifacts, making it possible for the nodular volume dynamic enhanced scanning.

Benign and malignant SPN cases exhibited differential characteristics on CT imaging. In the present study, our results showed that, the main CT signs for malignant SPN cases included the lobulation, spicular sign, pleural indentation, and vacuole sign, with the incidences of $63.46,59.62,53.85$, and $25.00 \%$, respectively, which reflected the pathological changes for the lung tumors. Boundaries of malignant SPN lesions are mostly irregular or lobulated, which are associated with the different growth rate of each tumor part, as well as the surrounding structure restriction. In particular, deep lobulation in the malignant SPN lesions has great diagnostic value (1). In the present study, the incidence of lobulation was $63.46 \%$ for the malignant SPN group. In line with this, a previous study has shown that the incidence of lobulation for the malignant SPN cases (64.40\%) is significantly higher than the benign SPN cases (9).

Moreover, spicular sign is a result of the extension and infiltration of tumor tissues into the adjacent lung parenchyma, which has been commonly seen in malignant nodules. A previous study has shown that the incidence of spicular sign 
Table II. Comparison of the morphological signs between benign and malignant SPN cases.

\begin{tabular}{lccrr}
\hline & Malignant SPN $(\mathrm{n}=52), \mathrm{n}(\%)$ & Benign SPN (n=41), n (\%) & $\chi^{2}$ & P-value \\
\hline Spicular sign & $31(59.62)$ & $7(17.07)$ & 22.115 & $<0.01$ \\
Lobulation & $33(63.46)$ & $8(19.51)$ & 23.804 & $<0.01$ \\
Pleural indentation & $28(53.85)$ & $6(21.43)$ & 18.988 & $<0.01$ \\
Vacuole sign & $13(25.00)$ & $2(4.88)$ & 7.752 & $<0.01$ \\
Aerial bronchogram & $6(11.54)$ & $4(9.76)$ & 0.098 & $<0.01$ \\
Vessel convergence & $9(17.31)$ & $7(17.07)$ & 0.413 & $<0.01$ \\
Satellite lesions & $2(3.84)$ & $2(4.88)$ & 0.375 & $<0.01$ \\
Calcification & $3(5.77)$ & $9(21.95)$ & 4.137 & $<0.01$ \\
\hline
\end{tabular}

for malignant SPN is $67.7 \%$, which represents a risk factor for malignant SPN (10). In the present study, the incidence of spicular sign for the malignant SPN group was $59.62 \%$. Vacuolar sign is induced by the tumor-damaged alveolar structures within the nodules. It has been shown that the incidence of vacuolar sign in the lung tumors is approximately $24-48 \%$, which has been mainly seen in the adenocarcinomas, squamous carcinomas, and bronchiolo-alveolar carcinomas (11). Our results for the incidence of vacuolar sign (25.00\%) were basically consistent with these findings. In addition, pleural indentation is another potent sign for the identification of benign and malignant SPN cases (12).

A previous study has shown that, in 29 cases of malignant nodules, there are 18 cases with pleural indentation, and the majority of remaining cases without pleural indentation have malignant nodules far away from pleura (13). Li et al (13) have shown that, the incidence of pleural indentation is $13.1 \%$ for the benign nodules, which is $25.4 \%$ for the malignant nodules. Moreover, Cui et al (14) have shown that, it is not specific to diagnose lung cancer with only pleural indentation, while the specificity for pleural indentation combined with associated notch (i.e., the notch connected to nodules) in diagnosing lung cancer is as high as $96 \%$, with the positive prediction rate of $97 \%$.

Air bronchogram refers to the presence of air bronch within SPN lesions. It has been shown that, the incidences of air bronchogram for benign and malignant nodules are 5.9 and $33 \%$, respectively. Ma et al (15) have shown that, the incidence of air bronchogram for the adenocarcinoma is as high as up to 48. $8 \%$, while the incidences for squamous carcinoma, alveolar carcinoma, and undifferentiated carcinoma were 20, 9.1, and $28.6 \%$, respectively.

Vessel convergence refers to the sign in which one or more vessels surrounding SPN were dragged by the lesions to accumulate around or pass through the lesions, or truncated by the boundaries. It has been shown that, malignant lesions are associated with truncation, uneven wall thickening, lumen expansion, and tail-like changes of adjacent bronchi. On the other hand, benign lesions usually compress and flatten the adjacent bronchi, without wall thickening (16). It has been previously shown that the incidences of vessel convergence for benign and malignant SPN are 12 and $31 \%$, respectively. However, in this study, no significant differences have been observed in the air bronchogram and vessel convergence between benign and malignant groups. This might be due to the limited sample size herein, which needed further in-depth studies.

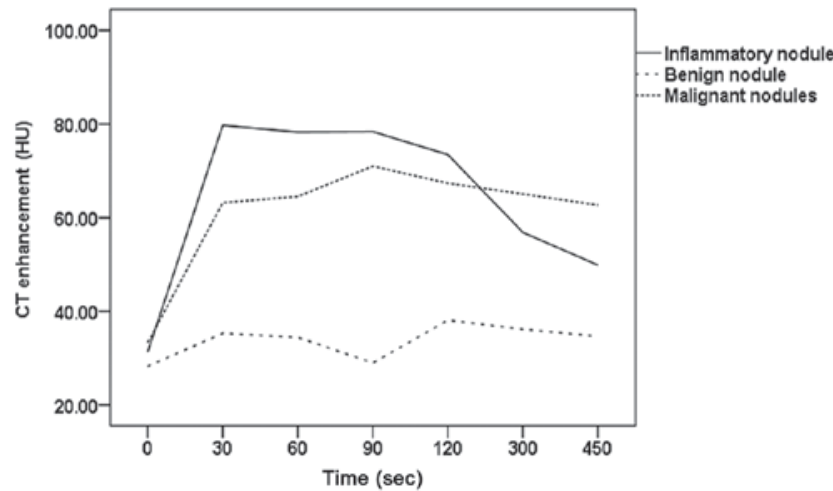

Figure 2. Dynamic enhancement of different SPN types. TDCs of malignant and benign SPN, as well as inflammatory nodules.

Our results showed that, the case number of calcification within benign SPN lesions is significantly higher than the malignant lesions. In the past, calcification has once been recognized as benign lesion (17). However, later studies have shown that, even SPN cases with calcification could not be excluded from malignant tumors (18). Fat in the SPN represents a strong evidence for benign lesions, which is commonly seen in hamartoma. Calcification and fat density could be present within SPN, which always imply benign lesions. At present, studies concerning SPN have been mainly focusing on single sign and the prediction model for benign and malignant lesions (19). However, in fact, there might be one or more signs in a case. Therefore, it is difficult to distinguish between benign and malignant lesions based on one single sign, and analysis of combined multiple signs is needed.

Due to the lack of typical signs such as lobulation and burr in some SPN cases, the imaging performance crossover with some benign nodules, making it difficult for the early diagnosis and treatment of cancers. Some patients with benign nodules receive surgical treatment before the clear diagnosis, which increases the patients' suffering, reduce their quality of life, and causes the waste of medical resources. MSCT dynamic enhancement is an important method to evaluate SPN (assessing hemodynamics and perfusion status), which measures densities at different time points after injection of contrast agent and provides evaluable evidence for differential diagnosis. A previous study performed enhancement detection on small nodules in lung, and they have found out that the enhancement peak values 
Table III. Comparison of dynamic enhancement CT values for benign an malignant SPN.

Dynamic enhancement time (s) Benign SPN (n=22), HU Malignant SPN (n=52), HU Inflammatory nodules (n=19), HU

\begin{tabular}{llll}
\hline 0 (routine) & $27.38 \pm 10.87$ & $33.69 \pm 7.21$ & $31.25 \pm 10.10$ \\
30 & $35.41 \pm 14.97$ & $63.95 \pm 11.32^{\mathrm{a}}$ & $79.13 \pm 23.62^{\mathrm{a}}$ \\
60 & $34.87 \pm 18.75$ & $64.57 \pm 10.21^{\mathrm{a}}$ & $78.37 \pm 21.57^{\mathrm{a}}$ \\
90 & $28.06 \pm 11.52$ & $71.33 \pm 11.08^{\mathrm{a}}$ & $78.95 \pm 26.21^{\mathrm{a}}$ \\
120 & $38.32 \pm 15.64$ & $67.89 \pm 10.14^{\mathrm{a}}$ & $73.95 \pm 20.35^{\mathrm{a}}$ \\
300 & $36.02 \pm 13.27$ & $65.13 \pm 9.22^{\mathrm{a}}$ & $57.22 \pm 28.63^{\mathrm{a}, \mathrm{b}}$ \\
540 & $34.81 \pm 13.95$ & $62.57 \pm 7.61^{\mathrm{a}}$ & $49.36 \pm 23.04^{\mathrm{a}, \mathrm{b}}$ \\
\hline
\end{tabular}

Compared with benign SPN, ${ }^{a} \mathrm{P}<0.01$; compared with malignant $\mathrm{SPN},{ }^{\mathrm{b}} \mathrm{P}<0.01$.

Table IV. Comparison of diagnostic accordance rates.

In accordance with surgical and puncture findings

\begin{tabular}{llllr}
\cline { 3 - 4 } Variables & Yes, n (\%) & No, n (\%) & $\chi^{2}$ & P-value \\
\hline Morphological signs & $66(70.97)$ & $27(29.03)$ & 6.257 & $<0.05$ \\
Morphological signs + dynamic enhancement & $78(83.87)$ & $15(16.13)$ & & \\
\hline
\end{tabular}
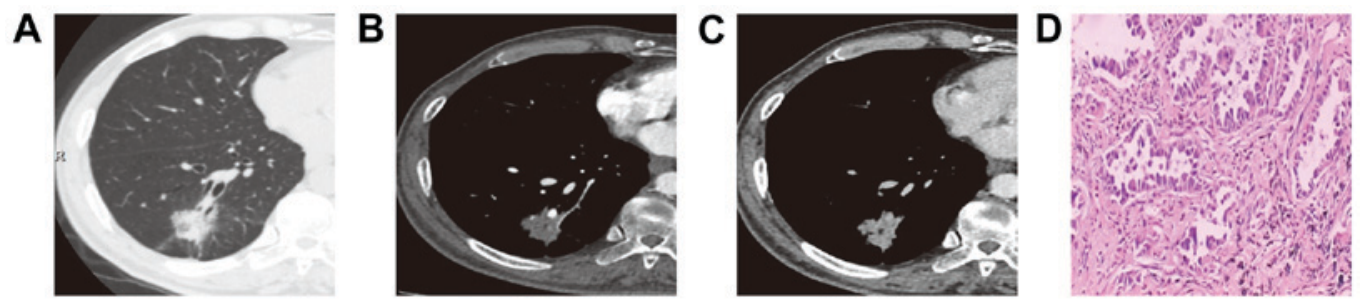

Figure 3. Dynamic enhancement CT scanning for malignant SPN cases. (A) Right lower lobe nodule. (B) Dynamic enhancement CT value at 30 sec after contrast injection was $59 \mathrm{HU}$. (C) Dynamic enhancement CT value at $300 \mathrm{sec}$ after contrast injection was $68 \mathrm{HU}$. (D) Adenocarcinoma as confirmed by postoperative pathology. Magnification, x20.
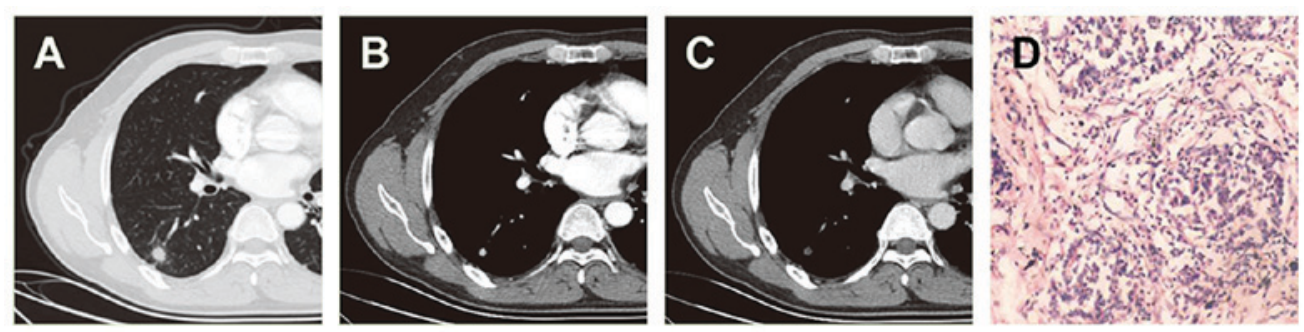

Figure 4. Dynamic enhancement CT scanning for inflammatory nodules. (A) Right lower lobe nodule, accompanied by halo sign and pleural indentation. (B) Dynamic enhancement CT value at $30 \mathrm{sec}$ after contrast injection was $81 \mathrm{HU}$. (C) Dynamic enhancement CT value at 300 sec after contrast injection was $46 \mathrm{HU}$. (D) Inflammatory nodules as confirmed by puncture biopsy pathology. Magnification, x10.

are different between benign and malignant nodules $(20,21)$. Appropriate peak enhancement threshold can help identifying the characteristics of SPN. They have shown that, CT values increase after enhancement of $<15 \mathrm{HU}$ indicate benign nodules, while CT values increase after enhancement of $>15$ HU suggest malignant nodules, with the sensitivity, specificity, and accuracy of 98,58 , and $77 \%$, respectively. Moreover, it has been also shown that, enhancement of $\leq 20 \mathrm{HU}$ or $\geq 60 \mathrm{HU}$ indicates benign nodules, while the enhancement of 20-60 HU suggests malignant nodules, and enhancement of $\geq 60 \mathrm{HU}$ is probably associated with active inflammatory nodules (22).

Jeong et $\mathrm{al}_{2}$ and Zhang and Kono $(23,24)$ have studied the time-density curve (TDC) of SPN. They have found that, for inflammatory SPN, the TDC increases rapidly, with the highest 
peak, after which the TDC shortly decreases and then increases, then followed by obvious decline. However, for benign SPN, the TDC is at a low level, or almost without elevation. Furthermore, for malignant SPN, the TDC increases rapidly, stays on plateau after peaking, and then slightly declines. In line with our results, different TDC morphologies for benign and malignant lesions reflect the differences in fluid volume outside nodular cells and dispersion rate of contrast agent. In this study, for the inflammatory nodules and malignant SPN, during the dynamic enhancement scanning, the CT values at each time points were all significantly higher than the benign SPN group. Comparing the dynamic enhancement CT values at different time points, our results showed that, the dynamic enhancement $\mathrm{CT}$ values at $30,60,90$, and $120 \mathrm{sec}$ were significantly elevated for both the inflammatory nodules and malignant SPN cases. However, no significant differences were observed between these two groups. On the other hand, at 300 and $540 \mathrm{sec}$, the dynamic enhancement CT values were significantly declined in the inflammatory nodules, while no obvious decline was observed for the malignant SPN group. The dynamic enhancement CT values at 300 and $480 \mathrm{sec}$ for the inflammatory nodules were significantly lower than the malignant SPN group. Based on these results, CT dynamic enhancement detection has great value for the identification of benign and malignant nodules. However, the differential findings from different studies might be due to different nodule natures, different parameters (such as the total amount of contrast agent, injection rate, and delayed scanning time), and some complex physiological factors (such as cardiac output, pulmonary blood flow, and body weight), which should be considered for the differential diagnosis. Clear and accurate exhibition and identification of morphological signs, combined with the dynamic enhancement detection, are of great clinical value for the differential diagnosis of benign and malignant nodules. Our results showed that, the diagnostic accordance rate for the morphological signs combined with dynamic enhancement diagnosis was significantly higher than the morphological signs alone.

In summary, the morphological features of SPN are of great significance and importance for the differential diagnosis in clinic. Dynamic enhancement CT scanning could provide a variety of dynamic enhancement parameters for nodules, which might be valuable for the identification of benign and malignant lesions. Comprehensive application of morphological features and dynamic enhancement detection can improve the diagnostic accuracy of malignant nodules, contributing to the disease early diagnosis, in time treatment, and prognosis, and avoiding the waste of medical resources due to unnecessary surgeries.

\section{Acknowledgements}

We are grateful to Jian Yang, Deputy Chief Physician from the Department of Radiology, Chongqing General Hospital for the assistance in the manuscript preparation.

\section{References}

1. Wahidi MM, Govert JA, Goudar RK, Gould MK and McCrory DC American College of Chest Physicians: Evidence for the treatment of patients with pulmonary nodules: When is it lung cancer? ACCP evidence-based clinical practice guidelines (2nd edition). Chest 132 (3 Suppl): 94S-107S, 2007.
2. Prakashini K, Babu S, Rajgopal KV and Kokila KR: Role of computer Aided diagnosis (CAD) in the detection of pulmonary nodules on 64 row multi detector computed tomography. Lung India 33: 391-397, 2016.

3. Lin JZ, Zhang L, Zhang CY, Yang L, Lou HN and Wang ZG: Application of gemstone spectral computed tomography imaging in the characterization of solitary pulmonary nodules: Preliminary result. J Comput Assist Tomogr 40: 907-911, 2016.

4. Truong MT, Sabloff BS and Ko JP: Multidetector CT of solitary pulmonary nodules. Radiol Clin North Am 48: 141-155, 2010.

5. Wu B: Comparison and analysis of diagnosis spiral CT and high-resolution CT for peripheral lung cancer with diameter of $<3 \mathrm{~cm}$. Chin Med Herald 8: 78-79, 2011 (In Chinese).

6. Wang B and Li H: Significance of dynamic contrast-enhanced CT in the differential diagnosis of solitary pulmonary nodules. Chin J Lab Diagn 16: 1495-1496, 2012 (In Chinese).

7. E LN and Ma D: Imaging diagnosis and management of small pulmonary nodules. Chin J Radio 43: 332-334, 2009 (In Chinese).

8. Shi Z, Wang Y and He X: Differential diagnosis of solitary pulmonary nodules with dual-source spiral computed tomography. Exp Ther Med 12: 1750-1754, 2016

9. He Q, Yu F, Dai P, Liu Z, Guo R and Yang B: Comparative study of MSCT and pathological findings of solitary pulmonary nodules. Chongqing Med 43: 3912-3915, 2014.

10. Yang D, Li Y, Liu J, Jiang G, Li J, Zhao H, Yang F, Liu Y, Zhou Z, Bu L and Wang J: Study on solitary pulmonary nodules: Correlation between diameter and clinical manifestation and pathological features. Zhongguo Fei Ai Za Zhi 13: 607-611, 2010 (In Chinese).

11. Macdonald K, Searle J and Lyburn I: The role of dual time point FDG PET imaging in the evaluation of solitary pulmonary nodules with an initial standard uptake value less than 2.5. Clin Radiol 66: 244-250, 2011.

12. Harders SW, Madsen HH, Rasmussen TR, Hager H and Rasmussen F: High resolution spiral CT for determining the malignant potential of solitary pulmonary nodules: Refining and testing the test. Acta Radiol 52: 401-409, 2011.

13. Li Y, Sui X and Yang D: Solitary pulmonary nodules: A risk factor analysis. Chin J Thoracic Cardiovasc Surg 26: 161-164, 2010 (In Chinese).

14. Cui Y, Ma D and Yang J: The value of pleural indentation in the diagnosis of pulmonary nodule: A meta-analysis. J Cap Med Univ 28: 709-712, 2007.

15. Ma YH, Li YX and Fang Y: A comparative study on pathology and CT signs of small peripheral lung cancer. Chin J Mod Med: 100-103, 2013 (In Chinese).

16. Zhao BY, Geng C and Beng BZ: The value of CT-vessel convergence sign in differential diagnosis of SPN. Chin Imaging Integr Trad West Med 4: 339-341, 2006 (In Chinese).

17. Khan A: ACR Appropriateness criteria on solitary pulmonary nodule. J Am Coll Radiol 4: 152-155, 2007.

18. Lin HL: Development in the diagnosis of solitary pulmonary nodule by CT. Med Recapitulate (Issue 11): 1725-1727, 2010 (In Chinese).

19. Li Y, Chen KZ, Sui XZ, Bu L, Zhou ZL, Yang F, Liu YG, Zhao H, Li JF, Liu J, et al: Establishment of a mathematical prediction model to evaluate the probability of malignancy or benign in patients with solitary pulmonary nodules. Beijing Da Xue Xue Bao Yi Xue Ban 43: 450-454, 2011 (In Chinese).

20. Kim JH, Kim HJ,Lee KH, Kim KH and Lee HL: Solitary pulmonary nodules: A comparative study evaluated with contrast-enhanced dynamic MR imaging and CT. J Comput Assist Tomogr 28: 766-775, 2004.

21. Swensen SJ, Viggiano RW, Midthun DE, Müller NL, Sherrick A, Yamashita K, Naidich DP, Patz EF, Hartman TE, Muhm JR and Weaver AL: Lung nodule enhancement at CT: Multicenter study. Radiology 214: 73-80, 2000.

22. Zhang M, Hua Z and Yu Z: Quantitative investigation of solitary pulmonary nodules with dynamic contrast-enhanced functional CT. Clin Oncol Cancer Res 1: 229-235, 2004.

23. Jeong YJ, Lee KS, Jeong SY,Chung MJ, Shim SS, Kim H, Kwon OJ and Kim S: Solitary pulmonary nodule: Characterization with combined wash-in and washout features at dynamic multi-detector row CT. Radiology 237: 675-683, 2005.

24. Zhang M and Kono M: Solitary pulmonary nodules: Evaluation of blood flow patterns with dynamic CT. Radiology 205: 471-478, 1997.

This work is licensed under a Creative Commons Attribution-NonCommercial-NoDerivatives 4.0 International (CC BY-NC-ND 4.0) License. 